\title{
Human African trypanosomiasis in travellers to Kenya
}

F Gobbi (federico.gobbi@sacrocuore.it) ${ }^{1}$, Z Bisoffi ${ }^{1}$

1. Centro per le Malattie tropicali, Ospedale Sacro Cuore-Don Calabria, Negrar, Verona, Italy

Citation style for this article:

Gobbi F, Bisoffi Z. Human African trypanosomiasis in travellers to Kenya.

Euro Surveill. 2012;17(10):pii=20109. Available online: http://www.eurosurveillance.org/ViewArticle.aspx?Articleld=20109

In this issue, two cases are described of human African trypanosomiasis (HAT) due to Trypanosoma brucei rhodesiense. They occurred recently in European tourists returning from Masai Mara area, Kenya, to Germany and Belgium, respectively $[1,2]$. These are, to our knowledge, the first two HAT cases described in travellers to Kenya in the last 12 years, while several cases were reported mainly from Tanzania (Serengeti and Tarangire game parks), Zambia, Zimbabwe and Malawi [3]. HAT, also known as sleeping sickness, is caused by a flagellated trypanosome protozoan, which is transmitted by Glossina (tsetse) flies. T. b. gambiense - found in western and central Africa - is transmitted mainly by G. palpalis, which prefers areas of vegetation near rivers and cultivated fields; $T$. $b$. rhodesiense - found in eastern and southern Africa - is transmitted predominantly by $G$. morsitans, which feeds on wild animals in savannah areas, far from human settlements [4]. While humans are the only substantial reservoir of $T . b . \mathrm{gam}$ biense, $T$. $b$. rhodesiense HAT is a zoonosis and humans occasionally visiting affected areas (usually for hunting or tourism) are accidental hosts. T. b. gambiense HAT is usually characterised by a chronic course of illness, lasting months to years, whereas $T$. $b$. rhodesiense HAT causes a more acute and aggressive course, clinically resembling acute septicaemia or severe falciparum malaria, with death occurring within days, weeks or months of the untreated disease. HAT of both forms is characterised by two distinct phases: the early or haemo-lymphatic stage and the late or meningoencephalitic stage, with trypanosome invasion of the central nervous system of patients surviving the early stage [5].

Although T. b. gambiense accounts for more than $90 \%$ of all reported cases of HAT worldwide, $T$. $b$. rhodesiense has been the cause of most imported cases [6]. From 2000 to 2010, there were reports of 94 HAT cases diagnosed in non- endemic countries, of which $72 \%$ were due to T. b. rhodesiense: of them, $82 \%$ were diagnosed at the first stage. For comparison, among 26 cases of T. b. gambiense HAT cases, $77 \%$ were diagnosed at the second stage [3]. The diagnosis of HAT requires demonstration of the parasite in peripheral blood, lymph node aspirate or cerebrospinal fluid. Cerebrospinal fluid examination is mandatory for the disease staging, even in the absence of neurological signs, particularly because the treatment of the two stages is different [7]: for $T$. $b$. rhodesiense HAT, suramin is the drug of choice in the first stage and melarsoprol (both are highly toxic drugs) in the second, while for T. b. gambiense, pentamidine is used in the first stage, a combination of nifurtimox and eflornithine in the second [8]. Serology is commercially available for $T$. b. gambiense only and PCR has not yet come into routine use.

The occurrence of two imported cases of $T . b$. rhodesiense HAT, who were returning from the Masai Mara area, in south-west Kenya, is not really surprising, considering that several cases were reported in the last decade from the Serengeti Park in north-west Tanzania. Although located in two different countries, the two parks constitute a single geographical entity, artificially divided by the Kenyan-Tanzanian border. Up until now, transmission of the parasite occurred sporadically in the southern part (Serengeti) and seems to have now extended northward, probably following migration of infected game. The whole area should therefore be considered at potential risk. In 2001, the almost simultaneous occurrence of HAT in two Italian patients returning from Tarangire and Serengeti national parks was promptly reported to ProMED and to the European Network for Tropical Medicine and Travel Health (TropNet), allowing the detection of a cluster of several cases occurring in a short space of time in tourists who had been in the same locations [9]. The importance of networks in Europe, such as the European Travel Medicine Network (EuroTravNet) and TropNet, to detect rare diseases and to disseminate the relevant information, cannot be overemphasised. Besides offering advice on travel and prevention measures, such networks are also crucial for the local public health system in endemic countries, where tourism in the parks represents a fundamental income. For example, in 2001, after the alert was issued, surveillance of domestic cattle in the Serengeti and Tarangire areas was conducted by the chief veterinary officer in order to ascertain if they might have played a role in transmission of the parasite to humans [9]. 
Awareness of HAT is an essential prerequisite to prompt diagnosis and disease management, thus avoiding the potentially fatal complications of the disease [3]. For every patient coming from Sub-Saharian Africa, HAT, although rare, must be included in the differential diagnosis of any febrile patient returning from areas at potential risk. Patients often recall tsetse bites but this is not always the case as for example in the recent German case. Urech et al., in a review of the published cases [5], reported the presence of fever in the vast majority of cases of HAT due to $T . b$. rhodesiense (98\%) and T. b. gambiense (93\%). A trypanosomal chancre, which consists of a tender, purplish, indurated area that develops at the site of the tsetse fly bite [7], is a very important clue, occurring more frequently in $T$. b. rhodesiense disease ( $84 \%$ versus $47 \%$ in T.b. gambiense HAT). While its presence is virtually pathognomonic, its absence should not exclude the disease. Gastrointestinal and hepatic symptoms such as nausea, vomiting or jaundice are not rare in travellers infected with T.b. rhodesiense HAT and could mislead the physician to a gastrointestinal infection [5]. In HAT patients, cardiac involvement with typical ECG alterations, as seen in the German case, is frequent. HAT cardiomyopathy generally subsides with treatment [10].

Even in the absence of any accompanying symptoms, a fever in a patient coming from Sub-Saharan Africa should prompt all clinicians to exclude malaria. If a thick blood smear is used for this, $T$. $b$. rhodesiense infection should not be missed, if present, as the sensitivity of a thick smear is high in the acute phase of the disease [3]. However, in $11 \%$ of travellers infected with $T$. b. rhodesiense, trypanosomes could not be detected in the first blood smear and repeated blood examinations were necessary [5]. An excessive reliance on malaria rapid diagnostic tests - which are increasingly suggested as a useful diagnostic tool, especially outside specialised, referral centres - might lead to T. b. rhodesiense HAT cases being missed, as well as other conditions such as relapsing fever caused by Borrelia. In the German case the reason why HAT was not diagnosed on presentation could be that malaria thin smear only was initially performed at the local hospital, which is a frequent practice in non-specialised centers, without doing the more sensitive thick smear. Whatever the reason, we argue that all travellers (including people who are long-term residents abroad and migrants) should have access to specialised (clinical and diagnostic) management if presenting with fever or other relevant symptoms This is even more important for the gambiense form of the disease, for which diagnosis is often more problematic. Moreover, while $T$. b. rhodesiense HAT cases have generally been tourists who have relatively easy access to appropriate healthcare, $T$. b. gambiense HAT outside endemic countries is typically observed in people who have been long-term residents overseas for missionary or work-related reasons or in migrants or refugees from endemic countries, including undocumented migrants who may have limited access to healthcare in the host country [3]. Clinical networks such as TropNet, with its vast experience from its 62 centres spread over Europe, can also offer advice and support for diagnosis and management of HAT.

As far as treatment is concerned, distribution of HAT drugs is the exclusive responsibility of the World Health Organization (WHO), as, except for pentamidine, they cannot be obtained on the market. To treat patients with imported HAT, hospital pharmacy services have to request drugs from WHO and provide patient data. The drugs are then received from WHO within 24 and 48 hours. However, to enable prompt start of treatment - which is particularly important for the acute rhodesiense disease - a few hospitals have requested and have been given anti-trypanosomal drugs and thus are repositories of these drugs [3]. Ideally, at least one such repository should be present in every European country, in order to avoid unnecessary delay in drug procurement, which can also arise due to custom procedures [11]. For some patients with first-stage $T$. b. rhodesiense HAT, treatment was initiated with the more readily available pentamidine, switching to suramin upon availability $[7,12]$.

As no vaccination is available, travellers to HATendemic areas should be alerted of this important albeit low risk and take general protective precautions [12]. The tsetse fly is active during daytime and is particularly attracted by motion and blue and black surfaces [13]. The patient reported in Germany used insect repellents, but wore shorts and short-sleeved shirts, while for the Belgian patient, this information is lacking $[1,2]$. Bites can be prevented by wearing wrist- and ankle-length clothing of thick material and avoiding dark-coloured clothing [6]. The fly is able to bite through thinly woven fabric: therefore the impregnation of clothing with permethrin is recommended, along with the application of a skin repellent [14].

These measures should be particularly kept in mind now that transmission has recently occurred, and more cases might be expected. Moreover, all referral centres for imported tropical diseases should stay alert and any new case should be promptly reported to the concerned networks, as this would concur to a better knowledge of the local situation. The authors of the German paper report that the local authorities in Kenya have been duly informed and that a WHO team of experts has been sent to the area, therefore we hope to receive further information in the coming weeks.

Acknowledgments

We are grateful to professors Johannes A. Blum, Christoph Hatz and August Stich for critical reading of the manuscript and useful inputs.

References 
1. Wolf T, Wichelhaus T, Göttig S, Kleine C, Brodt HR, JustNuebling $\mathrm{G}$. Trypanosoma brucei rhodesiense infection in a German traveller returning from the Masai Mara area, Kenya, January 2012. Euro Surveill. 2012;17(10):pii=20114. Available from: http://www.eurosurveillance.org/ViewArticle. aspx?Articleld $=20114$

2. Clerinx J, Vlieghe E, Asselman V, Van de Casteele S, Maes MB, Lejon V. Human African trypanosomiasis in a Belgian traveller returning from the Masai Mara area, Kenya, February 2012. Euro Surveill. 2012;17(10):pii=20111. Available online: http:// www.eurosurveillance.org/ViewArticle.aspx?Articleld=20111

3. Simarro PP, Franco JR, Cecchi G, Paone M, Diarra A, Ruiz Postigo JA, et al. Human African trypanosomiasis in nonendemic countries (2000-2010). J Travel Med. 2012;19(1):44-53.

4. Brun R, Blum J, Chappuis F, Burri C. Human African trypanosomiasis. Lancet. 2010. 375(9709):148-59.

5. Urech K, Neumayr A, Blum J. Sleeping sickness in travelers - do they really sleep?. PLoS Negl Trop Dis. 2011;5(11):e1358.

6. Gautret $P$, Clerinx J, Caumes $E$, Simon $F$, Jensenius $M$, Loutan L, et al. Imported human African trypanosomiasis in Europe, 2005-2009. Euro Surveill. 2009;14(36):pii=19327. Available from: http://www.eurosurveillance.org/ViewArticle. aspx?Articleld $=19327$

7. Blum JA, Neumayr AL, Hatz CF. Human African trypanosomiasis in endemic populations and travellers. Eur J Clin Microbiol Infect Dis. 2011 Sep 7. [Epub ahead of print].

8. Priotto G, Kasparian S, Mutombo W, Ngouama D, Ghorashian $\mathrm{S}$, Arnold U, et al. Nifurtimox-eflornithine combination therapy for second-stage African Trypanosoma brucei gambiense trypanosomiasis: a multicentre, randomised, phase III, noninferiority trial. Lancet. 2009;374(9683):56-64.

9. Jelinek T, Bisoffi Z, Bonazzi L, van Thiel P, Bronner U, de Frey A, et al. Cluster of African trypanosomiasis in travelers to Tanzanian national parks. Emerg Infect Dis. 2002;8(6):634-5.

10. Blum JA, Zellweger MJ, Burri C, Hatz C. Cardiac involvement in African and American trypanosomiasis. Lancet Infect Dis. 2008;8(10):631-41.

11. Bisoffi Z, Beltrame A, Monteiro G, Arzese A, Marocco S, Rorato $\mathrm{G}$, et al. African trypanosomiasis gambiense, Italy. Emerg Infect Dis. 11(11):1745-7.

12. Ripamonti D, Massari M, Arici C, Gabbi E, Farina C, Brini M, et al. African sleeping sickness in tourists returning from Tanzania: the first 2 Italian cases from a small outbreak among European travelers. Clin Infect Dis. 2002;34(1):E18-22.

13. Steverding D, Troscianko T. On the role of blue shadows in the visual behaviour of tsetse flies. Proc Biol Sci. 2004;271 Suppl 3: $\mathrm{S} 16-17$.

14. Sholdt LL, Schreck CE, Mwangelwa MI, Nondo J, Siachinji VJ. Evaluations of permethrin-impregnated clothing and three topical repellent formulations of deet against tsetse flies in Zambia. Med Vet Entomol. 1989;3(2):153-8. 